Research Paper

\title{
Risk factors of extra-hepatic progression after transarterial chemoembolization for hepatocellular carcinoma patients: a retrospective study in 654 cases
}

\author{
Shaohua Li $i^{1,5,6^{*}}$, Qiaoxuan Wang 2,5,6* Jie Mei1,5,6* Jianwei Wang $3,5,6$, Xiao-Ping Zhong7, Yihong Ling $4,5,6$, \\ Zhixing Guo ${ }^{3,5,6}$, Liang-He Lu ${ }^{1,5,6}$, Wei Wei ${ }^{1,5,6 凶}$, Rongping Guo ${ }^{1,5,6}$
}

1. Department of Hepatobiliary Oncology of the Sun Yat-sen University Cancer Center; Guangzhou 510060, P.R. China

2. Department of Radiation Oncology of the Sun Yat-sen University Cancer Center, Guangzhou 510060, P.R. China

3. Department of Ultrasound of the Sun Yat-sen University Cancer Center, Guangzhou 510060, P.R. China

4. Department of pathology of the Sun Yat-sen University Cancer Center, Guangzhou 510060, P.R. China

5. State Key Laboratory of Oncology in South China, Guangzhou 510060, P.R. China

6. Collaborative Innovation Center for Cancer Medicine, Guangzhou 510060, P.R. China

7. Department of Burn and Plastic Surgery, 2nd Affiliated Hospital of Shantou University Medical College, Shantou 515041, China

*These authors equally contributed to this study.

Corresponding authors: Rong-Ping Guo, M.D. and Wei Wei, M.D. Department of Hepatobiliary Oncology, Cancer Center, Sun Yat-sen University, Guangzhou, 510060, P.R. China. Telephone: (8620)-87342266; Fax: (8620)-87342266; Email: guorp@sysucc.org.cn.

(c) The author(s). This is an open access article distributed under the terms of the Creative Commons Attribution License (https://creativecommons.org/licenses/by/4.0/). See http://ivyspring.com/terms for full terms and conditions.

Received: 2019.03.31; Accepted: 2019.07.13; Published: 2019.08.27

\begin{abstract}
Aim: To investigate the risk factors of extra-hepatic progression after TACE in HCC.

Methods: The study population included $654 \mathrm{HCC}$ patients who underwent TACE between October 2005 and September 2012. We collected and analyzed their clinical characteristics and survival information. TACE was performed as previously described with minor modifications. When necessary, superselective chemoembolization was performed through the segmental or subsegmental arteries, based on the tumor location and extent and hepatic function reserve. If stasis could not be achieved in a tumor-feeding artery, iodized oil was used solely in some patients. Embolization was then performed with injection of absorbable gelfoam particles (1-2 mm in diameter) through the angiographic catheter.

Results: The tumor response to initial TACE was evaluated in 645 patients. The CR rate, response rate (RR), and disease control rate (DCR) were $9.92 \%, 25.89 \%$, and $70.39 \%$, respectively. The median overall survival (OS) period was 14.5 months. The 6-month, 1-, 2-, 3-, and 5-year OS rates were $75.5 \%, 55.0 \%, 33.9 \%, 22.8 \%$, and 14.9\%, respectively. The median progression-free survival (PFS) period was 4.3 months. The 6-month, 1-, 2-, 3-, and 5 -year PFS rates were $40.7 \%, 27.1 \%, 16.7 \%, 13.9 \%$, and $9.3 \%$, respectively. One hundred and fifty patients developed extrahepatic progression during follow-up. We demonstrated that in the absence of radical treatment after initial TACE $(p<0.001)$, the presence of extrahepatic metastasis before initial TACE $(p<0.001)$, AST $>45 \mathrm{U} / \mathrm{L}(\mathrm{p}=0.024), A L B<35 \mathrm{~g} / \mathrm{L}(\mathrm{p}=0.012)$, and tumor response were evaluated as PD and SD after initial TACE $(p<0.001)$ and were found to be independent predictors of a poorer prognosis of extrahepatic PFS.

Conclusions: We identified risk factors for extrahepatic progression after TACE in HCC patients. Early combination treatment was strongly recommended in patients that met these risk factors.
\end{abstract}

Key words: hepatocellular carcinoma; transarterial chemoembolization; extrahepatic progression; risk factor

\section{Introduction}

Hepatocellular carcinoma (HCC) is one of the most commonly diagnosed cancers and causes of cancer mortality worldwide.[1,2] Fewer than $20 \%$ of HCC patients can receive curative therapies such as surgical resection, liver transplantation and ablative therapies due to advanced disease at presentation or poor liver functional reserve.[3] Transarterial chemoembolization (TACE) is a widely accepted treatment for unresectable or intermediate-stage HCCs.[4-6] However, their prognosis remains poor.[6, 
7] Extrahepatic progression following TACE is one of the important factors. However, very few studies have been reported to evaluate the risk factors for extrahepatic progression after TACE in HCC patients.

This retrospective study was designed to investigate the risk factors for extrahepatic progression after TACE in HCC patients in order to improve individualized treatment and promote precision treatment of these patients.

\section{Materials and Methods}

This research was approved by the institutional review board (IRB) of Sun Yat-sen University Cancer Center. The approval number is B2018-134-01. The experiments were carried out in accordance with the approved guidelines.

\section{Patients}

Between October 2005 and September 2012, 1635 patients were newly diagnosed with HCC and received TACE as primary treatment in our department. Of these, $654(40 \%)$ cases with complete medical records were included into the current retrospective study.

The diagnosis of HCC was based on the diagnostic criteria for HCC described in the American Association for the Study of the Liver (AASLD) guidelines.[5] Routine pretreatment examination included blood chemistry, serum tumor biomarker such as alpha-fetoprotein (AFP), chest radiography, ultrasonography, tri-phase contrast-enhanced helical computed tomography (CT), and/or contrastenhanced magnetic resonance (MR) imaging of the abdomen. Further investigations were performed whenever there was clinical suspicion of extrahepatic metastases. Liver function was evaluated based on the Child-Pugh classification system [8] and the indocyanine green (ICG) clearance test was performed routinely within three days before treatment. The selection criteria for the TACE procedure depended on the tumor location and extent, the liver function and the general condition of the patient.

\section{TACE procedure}

TACE was performed as previously described with minor modifications.[6] When necessary, superselective chemoembolization was performed through the segmental or subsegmental arteries, based on the tumor location and extent and hepatic function reserve. If stasis could not be achieved in a tumor-feeding artery, iodized oil was used solely in some patients. Embolization was then performed with injection of absorbable gelfoam particles (1-2 $\mathrm{mm}$ in diameter) through the angiographic catheter.

\section{Post-TACE care and follow-up}

The posttreatment care and follow-up were performed routinely in all patients. A serum AFP assay, liver function test, and abdominal ultrasonography or tri-phase contrast-enhanced helical CT were performed monthly during the first three months. Thereafter, the patients were followed every 2-3 months with radiology and serum examination. Further investigations were performed as needed.

The modified Response Evaluation Criteria in Solid Tumors (mRECIST) was used to evaluate tumor response [9]. The first evaluation was performed one month after TACE. Another TACE treatment was performed every 4-8 weeks until one of the following end points was reached: (1) complete devascularization of the tumor, (2) technical impossibility to embolize the residual tumors, (3) development of contraindications to embolization, and (4) total resection or ablation of the tumor by subsequent surgery or local ablation. The proper subsequent treatment was defined as the clinical routine. The follow-up ended on January 31, 2018.

\section{Statistical analysis}

Comparisons between categorical variables were performed using Pearson's $\chi 2$ test or Fisher's exact test where appropriate. Continuous variables were compared using Student's t-test (when values were normally distributed) or the Mann-Whitney test (when values had a distribution that departed significantly from normal). The survival analysis was calculated using the Kaplan-Meier method and compared using the log-rank test. Univariate and multivariate analyses using Cox' proportional hazard model were performed to evaluate the prognostic factors. The correlation between two variables was examined by Pearson's correlation analysis (when values were normally distributed) or Spearman's correlation analysis (when values had a distribution that departed significantly from normal). A value of $\mathrm{p}<0.05$ was considered statistically significant. All data were analyzed using SPSS statistical software for Windows (ver. 18.0; SPSS Inc., Chicago, IL, USA).

All continuous variable data are expressed as the mean \pm standard error (when values were normally distributed) or the median (range) (when values had a distribution that departed significantly from normal). All data regarding categorical variables are shown as $\mathrm{n}$ (proportion).

\section{Results}

\section{Baseline clinical characteristics}

The baseline clinical characteristics of 654 HCC patients treated with TACE are summarized in Table 
1. In the study cohort, the mean age was 51.23 years; most of the patients were male (603 cases, $92.20 \%)$ and hepatitis B surface antigen positive (576 cases, $88.07 \%$ ). Serum AFP level was higher than $400 \mathrm{ng} / \mathrm{ml}$ in approximately half of the patients (332 cases, $50.76 \%$ ). The liver function was good in most of the patients classified as Child-Pugh A (564 cases, 86.24\%) and B (85 cases, $13.00 \%$ ); the median ICG 15 minute retention (ICGR15) was 6.1\%. There were 249 cases (38.07\%) staged as stage A, 160 cases $(24.46 \%)$ as stage B, 243 cases $(37.16 \%)$ as stage $C$, and 2 cases $(0.31 \%)$ as stage $\mathrm{D}$, according to the BCLC algorithm. Seventy cases $(10.70 \%)$ had extrahepatic metastases, and 207 cases $(31.65 \%)$ had portal/hepatic vein tumor thrombosis.

Table 1. Baseline clinical characteristics and evaluation of tumor response after initial TACE in $654 \mathrm{HCC}$ patients

\begin{tabular}{|c|c|}
\hline Baseline clinical characteristics & $\begin{array}{l}\mathrm{n}=654 ; \text { mean } \pm \mathrm{SE} \text {, median (range), or } \mathrm{n} \\
\text { (proportion) }\end{array}$ \\
\hline Age (years) & $51.23 \pm 0.48$ \\
\hline \multicolumn{2}{|l|}{ Gender } \\
\hline Male & $603(92.20 \%)$ \\
\hline Female & $51(7.80 \%)$ \\
\hline \multicolumn{2}{|l|}{ HBsAg status } \\
\hline Negative & $65(9.94 \%)$ \\
\hline Positive & $576(88.07 \%)$ \\
\hline Unknown & $13(1.99 \%)$ \\
\hline \multicolumn{2}{|l|}{ Preoperative AFP level } \\
\hline$\leq 400 \mathrm{ng} / \mathrm{ml}$ & $322(49.24 \%)$ \\
\hline$>400 \mathrm{ng} / \mathrm{ml}$ & $332(50.76 \%)$ \\
\hline Preoperative ALT level (U/L) & $45.4(6 \sim 667.4)$ \\
\hline Preoperative AST level (U/L) & $57(13 \sim 521)$ \\
\hline $\begin{array}{l}\text { Preoperative TBil level } \\
\text { (umol/L) }\end{array}$ & $15.4(2.92 \sim 128)$ \\
\hline Preoperative ALB level (g/L) & $40.15 \pm 0.18$ \\
\hline Preoperative WBC level $\left(10^{9} / \mathrm{L}\right)$ & $6.6(2 \sim 27)$ \\
\hline Preoperative PLT level $\left(10^{9} / \mathrm{L}\right)$ & $176(16 \sim 594)$ \\
\hline Preoperative PT level (s) & $12.6(8.7 \sim 22.8)$ \\
\hline \multicolumn{2}{|l|}{ Preoperative Child-Pugh score } \\
\hline A5 & $333(50.92 \%)$ \\
\hline A6 & $231(35.32 \%)$ \\
\hline B7 & $63(9.63 \%)$ \\
\hline B8 & $18(2.75 \%)$ \\
\hline B9 & $4(0.61 \%)$ \\
\hline $\mathrm{C} 10$ & $2(0.31 \%)$ \\
\hline Unknown & $3(0.46 \%)$ \\
\hline Preoperative ICGR15 (\%) & $6.1(0 \sim 57.4)$ \\
\hline \multicolumn{2}{|l|}{ Number of tumors } \\
\hline Solitary & $399(61.01 \%)$ \\
\hline Multiple & $255(38.99 \%)$ \\
\hline $\begin{array}{l}\text { Maximum diameter of tumor } \\
(\mathrm{cm})\end{array}$ & $8.8(1 \sim 22)$ \\
\hline \multicolumn{2}{|l|}{ Extrahepatic metastasis } \\
\hline Negative & $584(89.30 \%)$ \\
\hline Positive & $70(10.70 \%)$ \\
\hline \multicolumn{2}{|c|}{ Portal/Hepatic vein tumor thrombosis } \\
\hline Negative & $447(68.35 \%)$ \\
\hline Positive & $207(31.65 \%)$ \\
\hline \multicolumn{2}{|l|}{ BCLC stage } \\
\hline Stage A & $249(38.07 \%)$ \\
\hline Stage B & $160(24.46 \%)$ \\
\hline Stage C & $243(37.16 \%)$ \\
\hline Stage D & $2(0.31 \%)$ \\
\hline Lipiodol dose (ml) & $15(0 \sim 70)$ \\
\hline \multicolumn{2}{|l|}{ Use of gelfoam } \\
\hline No & $543(83.03 \%)$ \\
\hline
\end{tabular}

\begin{tabular}{lc}
\hline Baseline clinical characteristics & $\begin{array}{l}\mathrm{n}=654 ; \text { mean } \pm \text { SE, median (range), or } \mathrm{n} \\
\text { (proportion) }\end{array}$ \\
\hline Yes & $111(16.97 \%)$ \\
Tumor response to initial TACE ( $\mathrm{n}=645)$ \\
CR & $64(9.92 \%)$ \\
PR & $103(15.97 \%)$ \\
SD & $287(44.50 \%)$ \\
PD & $191(29.61 \%)$ \\
Postoperative hospital stay & $5(1 \sim 105)$ \\
(days) & \\
\hline TACE: transcatheter arterial chemoembolization; HBsAg: hepatitis B surface \\
$\begin{array}{ll}\text { antigen; AFP: alpha fetoprotein; ALT: alanine aminotransferase; AST: aspartate } \\
\text { aminotransferase; TBIL: total bilirubin; ALB: albumin; WBC: white blood cell; PLT: }\end{array}$ \\
$\begin{array}{l}\text { blood platelet; PT: prothrombin time; BCLC: Barcelona Clinic Liver Cancer; CR: } \\
\text { complete response; PR: partial response; SD: stable disease; PD: progression disease }\end{array}$
\end{tabular}

\section{Tumor response and subsequent treatment}

The tumor response to initial TACE was evaluated in 645 patients, as shown in Table 1. The CR rate, response rate (RR), and disease control rate (DCR) were $9.92 \%, 25.89 \%$, and $70.39 \%$, respectively.

The subsequent treatment was chosen based on the tumor location and extent, the liver function and the general condition of the patient as shown in Table 2. Overall, 172 cases $(26.30 \%)$ received 342 cycles of radical treatments afterwards.

Table 2. The subsequent treatment following initial TACE of all patients

\begin{tabular}{lll}
\hline Treatment & Cases & Cycles \\
\hline Radical treatment & & \\
Resection for primary lesion & 46 & 49 \\
RFA for primary lesion & 85 & 121 \\
PMCT for primary lesion & 64 & 115 \\
PEI for primary lesion & 31 & 46 \\
Cryoablation for primary lesion & 6 & 8 \\
Transplantation & 3 & 3 \\
Palliative treatment & & \\
TACE & 323 & 555 \\
TAI & 9 & 10 \\
Systemic chemotherapy & 19 & 55 \\
Sorafenib & 45 & - \\
Radiotherapy & 18 & 19 \\
Sealed source radiotherapy & 7 & 13 \\
Resection for metastatic lesion & 1 & 1 \\
PMCT for metastatic lesion & 4 & 5 \\
RFA for metastatic lesion & 3 & 6 \\
Cryoablation for metastatic lesion & 1 & 1 \\
CIK cell therapy & 5 & 12 \\
\hline RFA: radiofrequency ablation; PMCT: percutaneous microwave tumor coagulation \\
therapy; PEI: percutaneous ethanol injection therapy; TACE: transcatheter arterial \\
chemoembolization; TAI: transarterial infusion chemotherapy; CIK: \\
cytokine-induced killer & \multicolumn{2}{l}{}
\end{tabular}

\section{Survival and progression}

At a median follow-up time of 12.7 months (range, 0.1-127), 532 patients $(81.35 \%)$ had died. Of these, 4 patients died without any radiographic progression, which were counted in extrahepatic progression as shown in Table 3 . The median overall survival (OS) period was 14.5 (95\% confidence interval (CI), 12.6-16.3) months. The overall survival is shown in Fig. 1. The 6-month, 1-, 2-, 3-, and 5-year OS rates were $75.5 \%, 55.0 \%, 33.9 \%, 22.8 \%$, and $14.9 \%$, 
respectively. During the follow-up period, 488 patients $(74.62 \%)$ had disease progression. The median progression-free survival (PFS) period was 4.3 (95\% CI, 3.6-5.0) months. The progression-free survival is shown in Fig. 2. The 6-month, 1-, 2-, 3-, and 5-year PFS rates were $40.7 \%, 27.1 \%, 16.7 \%, 13.9 \%$, and $9.3 \%$, respectively. Overall, 150 patients developed extrahepatic progression as shown in Table 3.

Table 3. The characteristics of 150 patients with extrahepatic progression

\begin{tabular}{ll}
\hline Site of extrahepatic progression & Cases \\
\hline Lung & 83 \\
Lymph node(s) & 24 \\
Bone & 13 \\
Lung+lymph node(s) & 8 \\
Adrenal gland(s) & 7 \\
Death & 4 \\
Bone+lymph node(s) & 2 \\
Lung+bone & 1 \\
Lung+adrenal gland+lymph nodes & 1 \\
Peritoneum & 1 \\
Chest wall & 1 \\
Lung+adrenal gland+chest wall & 1 \\
Peritoneum+bone+muscle & 1 \\
Lung+peritoneum+pleura & 1 \\
Peritoneum+lymph nodes & 1 \\
Adrenal gland+lymph nodes & 1
\end{tabular}

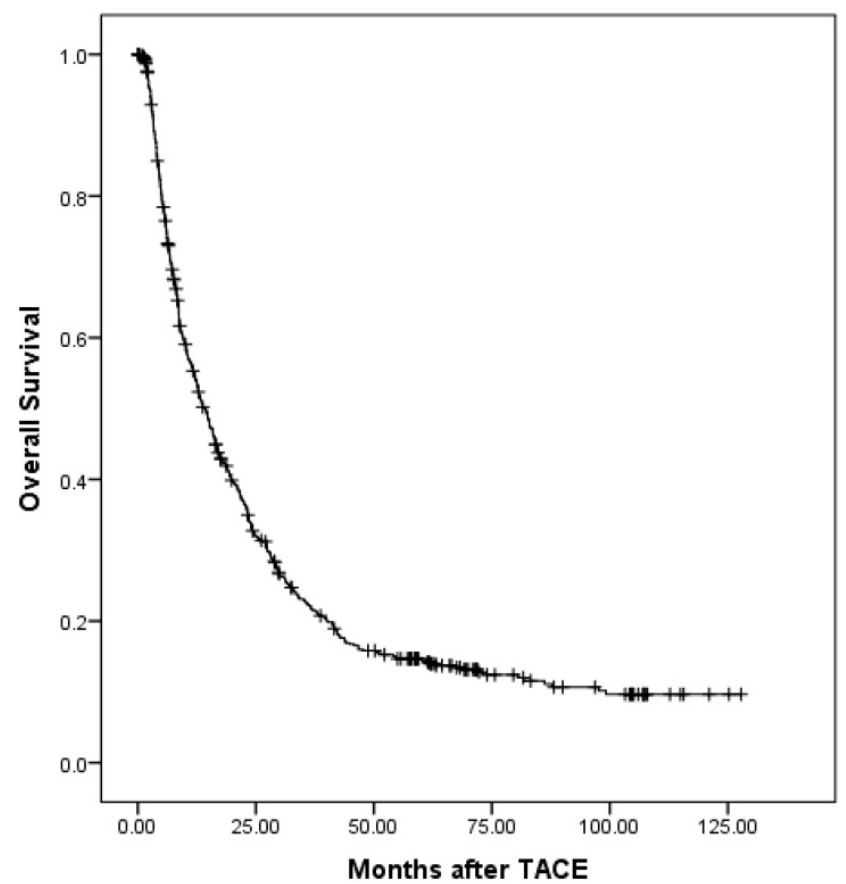

Figure 1. The overall survival of all patients.

\section{Prognostic factors for overall survival}

Univariate and multivariate analysis of factors affecting OS are shown in Table 4 . Age $\leq 50 \mathrm{yr}$, having not received radical treatment after initial TACE, a maximum tumor diameter $\geq 10 \mathrm{~cm}$, the presence of extrahepatic metastasis before initial TACE, the presence of portal/hepatic vein tumor thrombosis before initial TACE, AFP $>400 \mathrm{ng} / \mathrm{ml}$, platelet count $(\mathrm{PLT})>100 \times 109 / \mathrm{L}$, alanine aminotransferase (ALT) $>40 \mathrm{U} / \mathrm{L}$, aspartate transaminase $(\mathrm{AST})>45 \mathrm{U} / \mathrm{L}$, albumin (ALB) $<35 \mathrm{~g} / \mathrm{L}$, total bilirubin (TBil) $>20.5 \mu$ mol/L, Child-Pugh score $>6, \mathrm{~B} / \mathrm{C} / \mathrm{D}$ stage according to the BCLC staging system, lipiodol dose during TACE $>15 \mathrm{ml}$, the use of gelfoam during TACE, and tumor response that were evaluated as $\mathrm{PD}$ and $\mathrm{SD}$ after initial TACE and found to be statistically significant by univariate analysis were included in a multivariate regression analysis. The results of the latter demonstrated that not having received radical treatment after initial TACE ( $\mathrm{p}<0.001$, HR: 2.868; 95\% CI: 2.292-3.589), a maximum tumor diameter $\geq 10 \mathrm{~cm}$ ( $p=0.016$, HR: $1.327 ; 95 \%$ CI: 1.054-1.669), the presence of portal/hepatic vein tumor thrombosis $(\mathrm{p}=0.004$, HR: $1.427 ; 95 \%$ CI: 1.120-1.817), AFP $>400 \mathrm{ng} / \mathrm{ml}$ $(\mathrm{p}=0.001$, HR: $1.353 ; 95 \%$ CI: 1.125-1.629), AST >45 U/L ( $p=0.001$, HR: 1.534; 95\% CI: 1.200-1.960), and a tumor response that had been evaluated as PD and SD after initial TACE $(p<0.001$, HR: $1.829 ; 95 \%$ CI: 1.480-2.261) were independent predictors of poorer prognosis of OS.

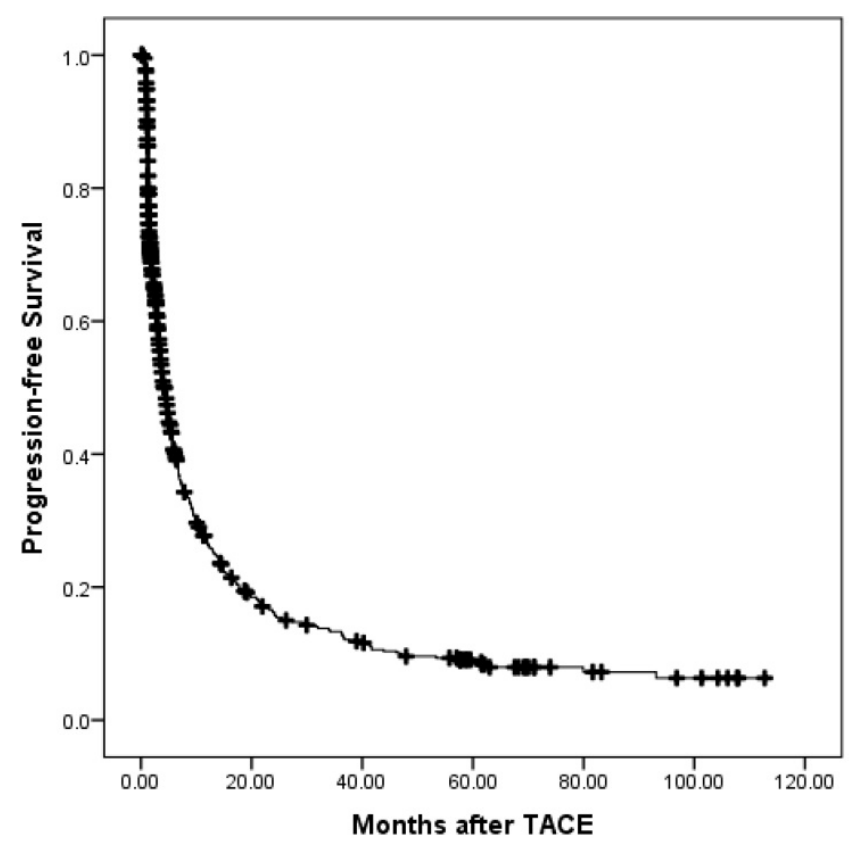

Figure 2. The progression-free survival of all patients.

\section{Prognostic factors for progression-free survival}

Similarly, univariate and multivariate analyses of factors affecting PFS are shown in Table 5. Not having received radical treatment after initial TACE, a maximum tumor diameter $\geq 10 \mathrm{~cm}$, the presence of extrahepatic metastasis before initial TACE, the presence of portal/hepatic vein tumor thrombosis before initial TACE, AFP $>400 \mathrm{ng} / \mathrm{ml}$, white blood cell count $($ WBC) $>4.0 \times 109 /$ L, PLT $>100 \times 109 /$ L, AST $>45$ 
U/L, ALB <35 g/L, Child-Pugh score >6, stage $\mathrm{B} / \mathrm{C} / \mathrm{D}$ according to the BCLC staging system, lipiodol dose during TACE $>15 \mathrm{ml}$, and tumor response that were evaluated as $\mathrm{PD}$ and $\mathrm{SD}$ after initial TACE and found to be statistically significant by univariate analysis were included in a multivariate regression analysis. The results of the latter revealed that not having received radical treatment after initial TACE ( $p<0.001$, HR: 1.663; 95\% CI: 1.347-2.053), portal/hepatic vein tumor thrombosis $(\mathrm{p}=0.021$, HR: 1.339; 95\% CI: 1.044-1.717), AST >45 U/L ( $p=0.024$, HR: 1.279; 95\% CI: 1.032-1.586), and tumor response that were evaluated as PD and SD after initial TACE $(p<0.001$, HR: 2.032; 95\% CI: 1.629-2.534) were independent predictors of poorer prognosis of PFS.

\section{Prognostic factors for extrahepatic progression-free survival}

Additionally, univariate and multivariate analyses of factors affecting extrahepatic PFS are shown in Table 6. Age $\leq 50 \mathrm{yr}$, not having received radical treatment after initial TACE, maximum tumor diameter $\geq 10 \mathrm{~cm}$, the presence of extrahepatic metastasis before initial TACE, the presence of portal/hepatic vein tumor thrombosis before initial TACE, AFP $>400 \mathrm{ng} / \mathrm{ml}$, PLT $>100 \times 109 / \mathrm{L}$, AST $>45$ $\mathrm{U} / \mathrm{L}, \mathrm{ALB}<35 \mathrm{~g} / \mathrm{L}$, stage $\mathrm{B} / \mathrm{C} / \mathrm{D}$ according to the $\mathrm{BCLC}$ staging system, lipiodol dose during TACE $>15$ $\mathrm{ml}$, and tumor response that were evaluated as PD and SD after initial TACE and found to be statistically significant by univariate analysis were included in a multivariate regression analysis. The results of the latter revealed that not having received radical treatment after initial TACE ( $\mathrm{p}<0.001$, HR: 2.629; 95\% CI: 1.697-4.072), the presence of extrahepatic metastasis before initial TACE $(\mathrm{p}<0.001, \mathrm{HR}$ : 2.259; 95\% CI: 1.432-3.563), AST >45 U/L $(\mathrm{p}=0.024, \mathrm{HR}$ : 1.612; 95\% CI: 1.065-2.439), ALB <35 g/L ( $p=0.012$, HR: 1.803; 95\% CI: 1.135-2.862), and tumor response that were evaluated as PD and SD after initial TACE ( $p<0.001$, HR: 2.608; 95\% CI: 1.670-4.071) were independent predictors of poorer prognosis of extrahepatic PFS.

\section{Discussion}

Although TACE has been widely used as a palliative therapy worldwide, especially in China and other Asian countries [6, 10-12], single TACE treatment is not recommended whenever extrahepatic metastasis is present [5, 13]. The BCLC staging classification recommends the administration of sorafenib as the first line treatment for HCC patients who have extrahepatic metastasis [5, 14]. A previous study showed that the prognosis of HCC patients with extrahepatic metastasis is significantly worse than that of advanced HCC patients without extrahepatic metastasis [15]. These results suggest that advanced HCC patients with extrahepatic metastasis or extrahepatic progression after TACE need combination treatments with TACE and systematic therapy, including sorafenib or radiotherapy [4], which makes identifying the risk factors for extrahepatic progression after TACE a matter of considerable importance.

Table 4. Univariate and multivariate analyses of factors affecting OS

\begin{tabular}{|c|c|c|c|c|c|c|}
\hline \multirow[b]{2}{*}{ Variables } & \multicolumn{3}{|c|}{ Univariate Cox regression analysis for OS } & \multicolumn{3}{|c|}{ Multivariate Cox regression analysis for OS } \\
\hline & HR & $95 \% \mathrm{CI}$ & $\mathrm{p}$ value & HR & $95 \% \mathrm{CI}$ & $\mathrm{p}$ value \\
\hline Male & 1.076 & $0.77-1.503$ & 0.668 & & & \\
\hline Age $(\leq 50$ yr $)$ & 1.261 & $1.063-1.496$ & 0.008 & 1.071 & $0.888-1.293$ & 0.472 \\
\hline No radical treatment & 3.146 & $2.54-3.895$ & 0.000 & 2.868 & $2.292-3.589$ & 0.000 \\
\hline Single lesion & 1.045 & $0.877-1.245$ & 0.623 & & & \\
\hline Maximum tumor diameter $(\geq 10 \mathrm{~cm})$ & 2.080 & $1.743-2.483$ & 0.000 & 1.327 & $1.054-1.669$ & 0.016 \\
\hline Extrahepatic metastasis present & 1.361 & $1.046-1.772$ & 0.022 & 0.945 & $0.707-1.263$ & 0.702 \\
\hline Portal/hepatic vein tumor thrombosis present & 2.323 & $1.933-2.791$ & 0.000 & 1.427 & $1.120-1.817$ & 0.004 \\
\hline $\mathrm{AFP}(>400 \mathrm{ng} / \mathrm{ml})$ & 1.677 & $1.412-1.992$ & 0.000 & 1.353 & $1.125-1.629$ & 0.001 \\
\hline $\mathrm{WBC}\left(>4.0 \times 10^{9} / \mathrm{L}\right)$ & 1.202 & $0.891-1.623$ & 0.228 & & - & \\
\hline $\operatorname{PLT}\left(>100 \times 10^{9} / \mathrm{L}\right)$ & 1.281 & $1.022-1.607$ & 0.032 & 1.090 & $0.841-1.413$ & 0.513 \\
\hline $\operatorname{ALT}(>40 \mathrm{U} / \mathrm{L})$ & 1.315 & $1.104-1.567$ & 0.002 & 0.956 & $0.77-1.186$ & 0.682 \\
\hline AST (>45 U/L) & 1.981 & $1.645-2.387$ & 0.000 & 1.534 & $1.200-1.960$ & 0.001 \\
\hline $\operatorname{ALB}(<35 \mathrm{~g} / \mathrm{L})$ & 1.338 & $1.043-1.717$ & 0.022 & 1.203 & $0.886-1.633$ & 0.237 \\
\hline TBil (>20.5 $\mu \mathrm{mol} / \mathrm{L})$ & 1.236 & $1.018-1.502$ & 0.033 & 1.022 & $0.813-1.284$ & 0.853 \\
\hline HBsAg positive & 1.154 & $0.868-1.534$ & 0.323 & & - & \\
\hline $\mathrm{PT}(>13.5 \mathrm{~s})$ & 1.142 & $0.942-1.384$ & 0.176 & & - & \\
\hline ICGR15 (>10\%) & 1.166 & $0.952-1.428$ & 0.138 & & - & \\
\hline Child-Pugh score $(>6)$ & 1.394 & $1.08-1.799$ & 0.011 & 1.240 & $0.88-1.747$ & 0.219 \\
\hline BCLC stage (B/C/D) & 1.436 & $1.202-1.717$ & 0.000 & 1.163 & $0.927-1.459$ & 0.191 \\
\hline Lipiodol dose (>15 ml) & 1.684 & $1.416-2.001$ & 0.000 & 0.953 & $0.772-1.177$ & 0.653 \\
\hline Use of gelfoam & 1.268 & $1.017-1.581$ & 0.035 & 1.054 & $0.822-1.351$ & 0.680 \\
\hline $\mathrm{PD}+\mathrm{SD}$ after initial TACE & 2.058 & $1.674-2.53$ & 0.000 & 1.829 & $1.480-2.261$ & 0.000 \\
\hline
\end{tabular}

OS: overall survival; AFP: alpha fetoprotein; WBC: white blood cell; PLT: blood platelet; ALT: alanine aminotransferase; AST: aspartate aminotransferase; TBIL: total bilirubin; ALB: albumin; HBsAg: hepatitis B surface antigen; PT: prothrombin time; ICG: indocyanine green; BCLC: Barcelona Clinic Liver Cancer; SD: stable disease 
Table 5. Univariate and multivariate analyses of factors affecting PFS

\begin{tabular}{|c|c|c|c|c|c|c|}
\hline \multirow[b]{2}{*}{ Variables } & \multicolumn{3}{|c|}{ Univariate Cox regression analysis for PFS } & \multicolumn{3}{|c|}{ Multivariate Cox regression analysis for PFS } \\
\hline & HR & $95 \% \mathrm{CI}$ & $\mathrm{p}$ value & HR & $95 \% \mathrm{CI}$ & $\mathrm{p}$ value \\
\hline Male & 1.279 & $0.902-1.814$ & 0.152 & & & \\
\hline Age $(\leq 50 \mathrm{yr})$ & 1.151 & $0.963-1.375$ & 0.122 & & & \\
\hline No radical treatment & 1.883 & $1.538-2.305$ & 0.000 & 1.663 & $1.347-2.053$ & 0.000 \\
\hline Multiple lesions & 1.015 & $0.847-1.217$ & 0.870 & & & \\
\hline Maximum tumor diameter $(\geq 10 \mathrm{~cm})$ & 1.507 & $1.25-1.818$ & 0.000 & 1.022 & $0.813-1.284$ & 0.855 \\
\hline Extrahepatic metastasis present & 1.501 & $1.125-2.004$ & 0.006 & 1.275 & $0.934-1.741$ & 0.126 \\
\hline Portal/hepatic vein tumor thrombosis present & 1.872 & $1.543-2.27$ & 0.000 & 1.339 & $1.044-1.717$ & 0.021 \\
\hline $\operatorname{AFP}(>400 \mathrm{ng} / \mathrm{ml})$ & 1.280 & $1.07-1.53$ & 0.007 & 1.081 & $0.893-1.308$ & 0.425 \\
\hline $\mathrm{WBC}\left(>4.0 \times 10^{9} / \mathrm{L}\right)$ & 1.543 & $1.107-2.149$ & 0.010 & 1.044 & $0.718-1.52$ & 0.821 \\
\hline PLT $\left(>100 \times 10^{9} / \mathrm{L}\right)$ & 1.335 & $1.052-1.693$ & 0.017 & 1.217 & $0.917-1.615$ & 0.174 \\
\hline $\operatorname{ALT}(>40 \mathrm{U} / \mathrm{L})$ & 1.146 & $0.956-1.375$ & 0.141 & & & \\
\hline AST (>45 U/L) & 1.503 & $1.244-1.815$ & 0.000 & 1.279 & $1.032-1.586$ & 0.024 \\
\hline $\operatorname{ALB}(<35 \mathrm{~g} / \mathrm{L})$ & 1.440 & $1.105-1.875$ & 0.007 & 1.260 & $0.918-1.73$ & 0.152 \\
\hline TBil (>20.5 umol/L) & 1.019 & $0.828-1.254$ & 0.861 & & & \\
\hline HBsAg negative & 1.007 & $0.753-1.347$ & 0.964 & & & \\
\hline $\mathrm{PT}(>13.5 \mathrm{~s})$ & 1.071 & $0.877-1.308$ & 0.501 & & & \\
\hline ICGR15 (>10\%) & 1.206 & $0.978-1.487$ & 0.080 & & & \\
\hline Child-Pugh score $(>6)$ & 1.330 & $1.015-1.741$ & 0.038 & 1.292 & $0.924-1.806$ & 0.134 \\
\hline BCLC stage $(B / C / D)$ & 1.370 & $1.139-1.647$ & 0.001 & 1.115 & $0.888-1.401$ & 0.347 \\
\hline Lipiodol dose (>15 ml) & 1.341 & $1.118-1.607$ & 0.002 & 0.987 & $0.795-1.226$ & 0.907 \\
\hline Use of gelfoam & 1.150 & $0.914-1.445$ & 0.233 & & & \\
\hline PD+SD after initial TACE & 2.202 & $1.78-2.725$ & 0.000 & 2.032 & $1.629-2.534$ & 0.000 \\
\hline
\end{tabular}

PFS: progression-free survival; AFP: alpha fetoprotein; WBC: white blood cell; PLT: blood platelet; ALT: alanine aminotransferase; AST: aspartate aminotransferase; TBIL: total bilirubin; ALB: albumin; HBsAg: hepatitis B surface antigen; PT: prothrombin time; ICG: indocyanine green; BCLC: Barcelona Clinic Liver Cancer; SD: stable disease

Table 6. Univariate and multivariate analyses of factors affecting extrahepatic PFS

\begin{tabular}{|c|c|c|c|c|c|c|}
\hline \multirow[b]{2}{*}{ Variables } & \multicolumn{3}{|c|}{ Univariate Cox regression analysis for extrahepatic progression-free survival } & \multicolumn{3}{|c|}{$\begin{array}{l}\text { Multivariate Cox regression analysis for } \\
\text { extrahepatic progression-free survival }\end{array}$} \\
\hline & HR & $95 \% \mathrm{CI}$ & $\mathrm{p}$ value & HR & $95 \% \mathrm{CI}$ & $\mathrm{p}$ value \\
\hline Male & 1.184 & $0.64-2.189$ & 0.591 & & & \\
\hline Age $(\leq 50 \mathrm{yr})$ & 1.723 & $1.245-2.385$ & 0.001 & 1.387 & $0.965-1.994$ & 0.077 \\
\hline No radical treatment & 3.093 & $2.027-4.72$ & 0.000 & 2.629 & $1.697-4.072$ & 0.000 \\
\hline Single lesion & 1.388 & $0.985-1.957$ & 0.061 & & & \\
\hline Maximum tumor diameter $(\geq 10 \mathrm{~cm})$ & 2.419 & $1.743-3.358$ & 0.000 & 1.143 & $0.76-1.719$ & 0.520 \\
\hline Extrahepatic metastasis present & 2.916 & $1.939-4.385$ & 0.000 & 2.259 & $1.432-3.563$ & 0.000 \\
\hline Portal/hepatic vein tumor thrombosis present & 2.577 & $1.85-3.589$ & 0.000 & 1.460 & $0.943-2.261$ & 0.090 \\
\hline $\mathrm{AFP}(>400 \mathrm{ng} / \mathrm{ml})$ & 1.770 & $1.276-2.454$ & 0.001 & 1.206 & $0.842-1.729$ & 0.307 \\
\hline WBC $\left(>4.0 \times 10^{9} / \mathrm{L}\right)$ & 1.477 & $0.819-2.667$ & 0.195 & & & \\
\hline PLT $\left(>100 \times 10^{9} / \mathrm{L}\right)$ & 1.808 & $1.117-2.927$ & 0.016 & 1.292 & $0.755-2.211$ & 0.350 \\
\hline $\operatorname{ALT}(>40 \mathrm{U} / \mathrm{L})$ & 1.323 & $0.947-1.85$ & 0.101 & & & \\
\hline $\mathrm{AST}(>45 \mathrm{U} / \mathrm{L})$ & 2.141 & $1.48-3.096$ & 0.000 & 1.612 & $1.065-2.439$ & 0.024 \\
\hline $\operatorname{ALB}(<35 \mathrm{~g} / \mathrm{L})$ & 1.839 & $1.194-2.832$ & 0.006 & 1.803 & $1.135-2.862$ & 0.012 \\
\hline TBil ( $\leq 20.5 \mathrm{umol} / \mathrm{L})$ & 1.033 & $0.707-1.51$ & 0.865 & & & \\
\hline HBsAg negative & 1.044 & $0.62-1.757$ & 0.871 & & & \\
\hline $\mathrm{PT}(\leq 13.5 \mathrm{~s})$ & 1.096 & $0.753-1.595$ & 0.632 & & & \\
\hline ICGR15 (>10\%) & 1.076 & $0.738-1.570$ & 0.703 & & & \\
\hline Child-Pugh score $(>6)$ & 1.546 & $0.981-2.436$ & 0.060 & & & \\
\hline BCLC stage $(B / C / D)$ & 1.544 & $1.099-2.168$ & 0.012 & 0.969 & $0.613-1.533$ & 0.894 \\
\hline Lipiodol dose $(>15 \mathrm{ml})$ & 1.848 & $1.338-2.554$ & 0.000 & 1.105 & $0.752-1.624$ & 0.611 \\
\hline Use of gelfoam & 1.150 & $0.763-1.735$ & 0.504 & & & \\
\hline PD+SD after 1st TACE & 2.984 & $1.945-4.576$ & 0.000 & 2.608 & $1.670-4.071$ & 0.000 \\
\hline
\end{tabular}

In the present study, several factors were found to be associated with OS and PFS. Some factors are well accepted in previous reports, such as tumor size, portal vein invasion, and AFP level [16-18]. Of note, while the tumor size, portal vein invasion, and AFP level were associated with OS and PFS, these factors were not significant predictors of extrahepatic progression in the present study, suggesting that in advanced HCC, the tumor burden in the liver itself is unrelated to extrahepatic progression after TACE treatment. It is controversial from the clinical point of view and numerous previous studies. In univariate analysis these factors are of significance. However, these may be obscured by other factors in the multivariate analysis. Although AFP can show good prognostic ability in most of the time, it is not an absolutely accurate prognostic indicator for extrahepatic progression. Tumor thrombi are meaningful in both multivariate analysis of OS and PFS, but show no significance in extrahepatic PFS. It may indicate that tumor thrombus is more likely to 
cause intrahepatic dissemination, rather than the progress of extrahepatic lesions in TACE treatment.

In the present study, the presence of extrahepatic metastasis did not affect OS and PFS, which is inconsistent with a previous study [15]. However, the presence of extrahepatic metastasis before TACE is an independent risk factor for extrahepatic progression. The results may be ascribed to the patients with extrahepatic metastasis suitable to accept combination treatment strategy such as systematic treatment and/or radiotherapy, which could enhance the anti-tumor effect compared to TACE alone.

Similarly, hypoproteinemia (albumin lower than $35 \mathrm{~g} / \mathrm{L}$ ) is a unique risk factor for extrahepatic progression, while other biomarkers for liver function such as total bilirubin, prothrombin time, ICGR15, alanine aminotransferase, and aspartate aminotransferase did not demonstrate prognostic prediction power for extrahepatic progression. This observation may suggest that albumin level plays quite a unique role in HCC patients, especially advanced patients. The treatment options of a significant number of patients may be limited by hypoproteinemia, making repeat TACE, combination therapy or other treatments impossible.

An increased AFP level has been associated with larger tumors and lower hypohepatia, reflecting an aggressive biology [19]. In present study, AFP $>400$ $\mathrm{ng} / \mathrm{ml}$ is associated with OS, which is consistent with a previous report [20]. However, no association was found with PFS and extrahepatic PFS, suggesting that TACE may inhibit the aggressive behavior of high AFP level tumors; this hypothesis needs further verification.

Of note, in the present study, extrahepatic metastasis present before TACE is an independent risk factors for extrahepatic progression after TACE, which is consistent with previous report [21]. Leal, et al. mentioned the patterns of progression were different between patients with and without extrahepatic metastasis. Based on the results of present and previous studies, we suggest apply combination therapy including target therapy and/or radiotherapy as early as possible in patients with extrahepatic metastasis.

We acknowledge some weaknesses in our study. First, the nature of retrospective study brought choose bias inevitably, the results needs further prospective study to confirm. Second, the chemotherapy regimen in TACE were various, which made the analysis more complex while making the results closer to real clinical practice at the same time in fact. Finally, to obtain the generalizability of our results, another validation cohort from other centers rather than our single center might be necessary.
In conclusion, we identified that the presence of extrahepatic metastasis before TACE, AST $>45 \mathrm{U} / \mathrm{L}$, ALB $<35 \mathrm{~g} / \mathrm{L}$, and lack of response after TACE as independent risk factors for extrahepatic progression. To gain better therapeutic outcome and survival, early combination treatment including target therapy and/or radiotherapy was strongly recommended in these patients.

\section{Acknowledgments}

This study was supported by the National Natural Science Foundation of China (No. 81871985), Natural Science Foundation of Guangdong Province (No. 2017A030310203), Medical Scientific Research Foundation of Guangdong Province (No. A2017477), Clinical Trials Project (5010 Project) of Sun Yat-Sen University (No. 5010-2017009), and Clinical Trials Project (308 Project) of Sun Yat-Sen University Cancer Center (No. 308-2015-014).

\section{Author Contributions}

LS, WQ, MJ contributed equally to this work; WW and GR also contributed equally to this work. LS, WW, and GR conceived and designed the experiments; LS, WQ, ZX, MJ, LL, and WW performed the experiments; LS, WQ, ZX, WJ, LY, GZ, and MJ analyzed the data; LS, WQ, ZX, and MJ contributed reagents, materials, and analysis tools; and LS, WQ, WJ, and WW wrote the paper. All authors have read and approved the final version of the manuscript.

\section{Institutional review board statement}

This study was approved by the institutional review board (IRB) of Sun Yat-sen University Cancer Center. The approval number is B2018-134-01. The experiments were carried out in accordance with the approved guidelines.

\section{Informed consent statement}

Patients were not required to give informed consent to the study because the analysis used anonymous clinical data that were obtained after each patient agreed verbally to undergo treatment. Individuals cannot be identified based on the data presented.

\section{Data sharing statement}

The study data are available from the Sun Yat-Sun University Cancer Center Institutional Data Access/Ethics Committee for researchers who meet the criteria for access to confidential data. Address: Sun Yat-Sun University Cancer Center Institutional Data Access, 651 Dongfeng East Road, Guangzhou 510060, P. R. China. E-mail: sfz@sysucc.org.cn. 


\section{Competing Interests}

The authors have declared that no competing interest exists.

\section{References}

1. Jemal A, Bray F, Center MM, Ferlay J, Ward E, Forman D. Global cancer statistics. CA Cancer J Clin. 2011; 61: 69-90.

2. El-Serag H, Rudolph K. Hepatocellular carcinoma: epidemiology and molecular carcinogenesis. Gastroenterology. 2007; 132: 2557-76.

3. Nishikawa H, Kita R, Kimura T, Osaki Y. Transcatheter arterial embolic therapies for hepatocellular carcinoma: a literature review. Anticancer research. 2014; 34: 6877-86.

4. Park J, Amarapurkar D, Chao Y, Chen P, Geschwind J, Goh K, et al. Consensus recommendations and review by an International Expert Panel on Interventions in Hepatocellular Carcinoma (EPOIHCC). Liver Int. 2013; 33: 327-37.

5. Bruix J, Sherman M, Practice Guidelines Committee AAftSoLD. Management of hepatocellular carcinoma. Hepatology. 2005; 42: 1208-36.

6. Luo J, Guo RP, Lai EC, Zhang YJ, Lau WY, Chen MS, et al. Transarterial chemoembolization for unresectable hepatocellular carcinoma with portal vein tumor thrombosis: a prospective comparative study. Annals of surgical oncology. 2011; 18: 413-20.

7. Natsuizaka M, Omura T, Akaike T, Kuwata Y, Yamazaki K, Sato T, et al. Clinical features of hepatocellular carcinoma with extrahepatic metastases. J Gastroenterol Hepatol. 2005; 20: 1781-7.

8. Pugh R, Murray-Lyon I, Dawson J, Pietroni M, Williams R. Transection of the oesophagus for bleeding oesophageal varices. Br J Surg. 1973; 60: 646-9.

9. Lencioni R, Llovet JM. Modified RECIST (mRECIST) assessment for hepatocellular carcinoma. Seminars in liver disease. 2010; 30: 52-60.

10. Xue $T$, Xie X, Zhang L, Yin X, Zhang B, Ren Z. Transarterial chemoembolization for hepatocellular carcinoma with portal vein tumor thrombus: a meta-analysis. BMC Gastroenterol. 2013; 13: doi: 10.1186/471-230X-13-60.

11. Lee H, Kim J, Choi I, Chung J, Park J, Kim C. The safety and efficacy of transcatheter arterial chemoembolization in the treatment of patients with hepatocellular carcinoma and main portal vein obstruction. A prospective controlled study. Cancer. 1997; 79: 2087-94

12. Yoo D, Kim K, Jin Y, Shim J, Ko G, Yoon H, et al. Clinical outcome of 251 patients with extrahepatic metastasis at initial diagnosis of hepatocellular carcinoma: does transarterial chemoembolization improve survival in these patients? J Gastroenterol Hepatol. 2011; 26: 145-54.

13. Verslype C, Rosmorduc O, Rougier P, ESMO Guidelines Working Group. Hepatocellular carcinoma: ESMO-ESDO Clinical Practice Guidelines for diagnosis, treatment and follow-up. Ann Oncol. 2012; 23: vii41-8.

14. Bruix J, Llovet J. Prognostic prediction and treatment strategy in hepatocellular carcinoma. Hepatology. 2002; 35: 519-24.

15. Yoo J, Lee J, Lee S, Lee M, Lee D, Cho Y, et al. Comparison of the effects of transarterial chemoembolization for advanced hepatocellular carcinoma between patients with and without extrahepatic metastases. PLoS One. 2014; 9: doi: 10.1371 /journal.pone. 0113926 .

16. Hu H, Kim J, Lee L, Kim K, Ko G, Yoon H, et al. Chemoembolization for hepatocellular carcinoma: multivariate analysis of predicting factors for tumor response and survival in a 362-patient cohort. J Vasc Interv Radiol. 2011; 22: 917-23.

17. Takayasu K, Arii S, Ikai I, Omata M, Okita K, Ichida T, et al. Prospective cohort study of transarterial chemoembolization for unresectable hepatocellular carcinoma in 8510 patients. Gastroenterology. 2006; 131: 461-9.

18. Memon K, Kulik L, Lewandowski R, Wang E, Riaz A, Ryu R, et al. Radiographic response to locoregional therapy in hepatocellular carcinoma predicts patient survival times. Gastroenterology. 2011; 141: 526-35.

19. Carr B, Guerra V, Giannini E, Farinati F, Ciccarese F, Rapaccini G, et al. Significance of platelet and AFP levels and liver function parameters for HCC size and survival. Int J Biol Markers. 2014; 29: e215-23.

20. Ichikawa T, Machida N, Sasaki H, Tenmoku A, Kaneko H, Negishi R, et al. Early Prediction of the Outcome Using Tumor Markers and mRECIST in Unresectable Hepatocellular Carcinoma Patients Who Underwent Transarterial Chemoembolization. Oncology. 2016; 91: 317-30.

21. Leal J, Gonen M, Covey A, Erinjeri J, Getrajdman G, Sofocleous C, et al. Locoregional Therapy for Hepatocellular Carcinoma with and without Extrahepatic Spread. J Vasc Interv Radiol. 2015; 26: 1112-21. 